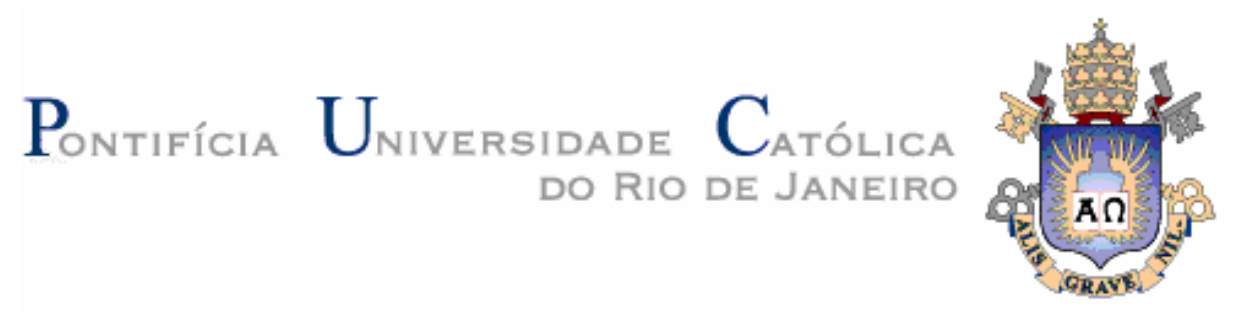

Liliane Tojeira Velozo

\title{
Metodização do Estudo das Fundações para Suportes de Linhas de Transmissão
}

Tese apresentada como requisito parcial para obtenção do título de Doutor pelo Programa de PósGraduação em Engenharia Civil da PUC-Rio.

Orientadoras: Deane de Mesquita Roehl Andréia Abreu Diniz de Almeida 


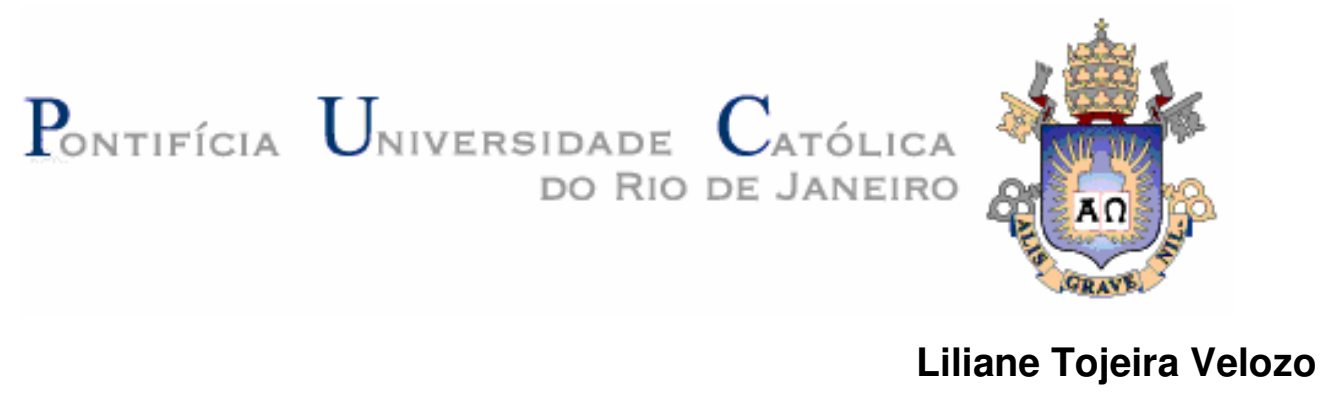

\title{
Metodização do Estudo das Fundações para Suportes de Linhas de Transmissão
}

\begin{abstract}
Tese apresentada como requisito parcial para obtenção do título de Doutor pelo Programa de Pós-Graduação em Engenharia Civil da PUC-Rio. Aprovada pela Comissão Examinadora abaixo assinada.
\end{abstract}

\author{
Deane de Mesquita Roehl \\ Orientadora \\ Departamento de Engenharia Civil - PUC-Rio \\ Andréia Abreu Diniz de Almeida \\ Co-orientadora \\ Departamento de Engenharia Civil - PUC-Rio \\ Paulo Gonçalves Batista \\ Departamento de Engenharia Civil - PUC-Rio \\ Elisa Dominguez Sotelino \\ Departamento de Engenharia Civil - PUC-Rio \\ João Luis Pascal Roehl \\ Consultor Independente \\ Nelson Henrique Costa Santiago \\ UFRJ \\ Prof. José Eugenio Leal
}

Coordenador Setorial do Centro Técnico Científico - PUC-Rio

Rio de Janeiro, 27 de maio de 2010 
Todos os direitos reservados. É proibida a reprodução total ou parcial do trabalho sem autorização da universidade, da autora e do orientador.

\section{Liliane Tojeira Velozo}

Graduou-se em Engenharia Civil pela Universidade Estadual do Rio de Janeiro em 2004. Mestre em Engenharia Civil com Ênfase em Estruturas pela Pontifícia Universidade Católica do Rio de Janeiro, PUC-Rio, em 2006. Iniciou o curso de doutorado na PUC-Rio em 2006.

Ficha Catalográfica

Tojeira Velozo, Liliane

Metodização do estudo das fundações para suportes de linhas de transmissão / Liliane Tojeira Velozo ; orientadoras: Deane de Mesquita Roehl, Andréia Abreu Diniz de Almeida. - 2010.

152 f. : il. (color.) ; $30 \mathrm{~cm}$

Tese (Doutorado)-Pontifícia Universidade Católica do Rio de Janeiro, Departamento de Engenharia Civil, Rio de Janeiro, 2010.

Inclui bibliografia

1. Engenharia civil - Teses. 2. Linhas aéreas de transmissão. 3. Fundações de LT's. 4. Confiabilidade de fundações. 5. Avaliação de risco. I. Roehl, Deane de Mesquita. I. II. Almeida, Andréia Abreu Diniz de. III. Pontifícia Universidade Católica do Rio de Janeiro. Departamento de Engenharia Civil. IV. Título. 
A minha avó Leonida, que sempre me incentivou a estudar, mesmo não tendo a mesma oportunidade.

Ao meu filho Pedro Augusto, que está a caminho e a quem desde já dedico minha vida e tudo ligado a ela. 


\section{Agradecimentos}

À Deus, por tudo que sou e tenho. A Ele toda Honra e toda a Glória;

A minha avó Leonida, que nesses últimos meses disse-me algumas vezes: "estou morta que acabes logo com isso";

Aos meus pais, Luiz e Luiza, e a meu irmão, Luiz Paulo, pela contribuição na minha formação como ser humano e pelo apoio em todas as minhas decisões;

Ao meu amor, Rafael Augusto, que tem contribuído para meu amadurecimento, pessoal e profissional, e para a formação de uma nova família;

A professora Deane que compreendeu minhas escolhas e incentivou o seguimento deste trabalho;

A professora Andréia pela disponibilidade e pela importante contribuição neste trabalho;

Ao professor João Luis Pascal Roehl, por todos os ensinamentos, técnico e de vida, a mim transmitidos. Obrigada pela compreensão e paciência;

Ao professor Jorge Martins Falcão, pela gentil colaboração, pelos materiais doados e emprestados e pelos conhecimentos técnicos compartilhados;

À Fluxo Engenharia, pela disponibilidade do suporte técnico necessário ao desenvolvimento desta tese de doutorado.

Ao CNPq, pelo apoio financeiro no início deste doutorado. 


\section{Resumo}

Velozo, Liliane Tojeira; Roehl, Deane de Mesquita; Almeida, Andréia Abreu Diniz de. Metodização do Estudo das Fundações para Suporte de Linhas de Transmissão. Rio de Janeiro, 2010. 143p. Tese de Doutorado Departamento de Engenharia Civil, Pontifícia Universidade Católica do Rio de Janeiro.

As fundações dos elementos de apoio de uma linha aérea de transmissão de energia elétrica situam-se ao longo de extensos traçados, onde diferentes condições de apoio para as estruturas são encontradas em razão da variação natural dos horizontes. Tal configuração pode levar alternativamente a se programarem estudos e sondagens especiais em cada local de suporte, fazendo crescer os custos e prazos de projeto, ou ao contrário, reduzindo drasticamente a investigação, com prejuízos evidentes ao planejamento, execução e economia da obra de implantação da linha de transmissão. Dessa maneira, são organizados e propostos procedimentos para avaliação do terreno segundo as suas orografia, hidrografia, geologia e pedologia e do ambiente atmosférico local, cujas manifestações são capazes de impor severas solicitações às torres e, indiretamente, às fundações. É eleita a região da bacia do rio da Prata para constituir o grande cenário do estudo, quer pela abundância de dados e informações disponíveis sobre linhas de transmissão já em carga, ou ainda em construção, nessa região. Assim sendo, desenvolve-se uma metodização para orientar o estudo dessas fundações, considerando o conjunto torre $\mathrm{x}$ terreno $\mathrm{x}$ fundação e buscando soluções técnicas convenientes e economicamente aceitáveis, atendendo assim ao binário desempenho-custo. Para as torres são considerados os modelos auto-suportados e estaiados, o terreno é variado entre algumas categorias identificadas na região e as fundações são representadas pelas superficiais, em sapatas, e pelas profundas do tipo tubulão. Buscando, ainda, auxiliar no aprimoramento dos projetos de fundações para suportes de linhas de transmissão, é desenvolvido um estudo de confiabilidade estrutural tendo como modelo algumas das fundações pré-selecionadas para a metodização primariamente desenvolvida. São determinadas as probabilidades de falha dessas fundações através do programa FERUM e, a partir do levantamento dos prejuízos financeiros causados pela falha desses elementos, determina-se o risco de falha. Por último, é desenvolvido um estudo paramétrico para avaliar a influência do 
projeto de fundação, do tipo de solo e da velocidade de vento sobre a probabilidade de falha.

\section{Palavras-chave}

1 - Linhas Aéreas de Transmissão; 2 - Fundações de LT's; 3 - Confiabilidade de Fundações; 4 - Avaliação de Risco. 


\section{Abstract}

Velozo, Liliane Tojeira; Roehl, Deane de Mesquita (Advisor); Almeida, Andréia Abreu Diniz de (Co-advisor). Methodization of Transmission Line Foundation - Study and Design. Rio de Janeiro, 2010. 143p. D.Sc. Thesis - Departamento de Engenharia Civil, Pontifícia Universidade Católica do Rio de Janeiro.

The transmission line tower foundations are executed following extensive paths, where different structural foundation conditions are found, in reason of natural horizontal ground layer variations. Then, one may alternatively to schedule and to execute special and specific studies or, conversely, to substantially reduce the geothecnical investigation, with sensible economical loses in the transmission line design and behavior. In such way, a methodology is proposed to evaluate conveniently the site according to its geology, orography, hydrography and pedology as well to related atmospheric local manifestations, able to submit severe mechanical solicitations to the towers and their foundations. In this way, one proposes a methodology to govern the study of these foundations, considering the tower $\mathrm{x}$ site $\mathrm{x}$ foundation relations and searching for convenient technical and acceptable economical solutions. Guyed and self-supported models are considered for towers, and the foundations are represented by footings, as the superficial type, and piers, as the deep type. The "rio do Prata" basin is chosen to constitute the large scenery of the study, because its abundant available data concerned to already operational transmission lines, or still under design and construction ones. Moreover, seeking the improvement of the tower foundation design a study based on structural reliability is developed considering some foundation models selected as mentioned above. Foundation failure probabilities are determined by the FERUM computational program and the risk of failure is obtained from economical damages caused by foundation failures. In the last, a parametric study is developed to evaluate how does the failure probability behave according to variations in the foundation soil, wind velocity and footing dimensions and depth.

\section{Keywords}

1 - Power Transmission Lines; 2 - TL's Foundations; 3 - Foundations Reliability; 4 - Risk Evaluation. 


\section{Sumário}

1 Introdução 19

1.1 Motivação 20

$\begin{array}{lll}1.2 \text { Objetivo } & 21\end{array}$

$\begin{array}{lll}1.3 & \text { Apresentação da Tese } & 21\end{array}$

2 Linhas de Transmissão na Região da Bacia do Prata 23

2.1 Situação Atual 23

2.1.1 Região Sul e Mato Grosso do Sul 24

2.1.2 São Paulo 25

2.1.3 Minas Gerais 26

2.1 .4 Goiás 26

2.2 Previsão de expansão com novas linhas de transmissão 27

3 Descrição Fisiográfica e Geológica da Região 28

$\begin{array}{lll}3.1 & \text { Hidrografia } & 28\end{array}$

3.2 Orografia 32

3.3 Geologia 36

3.3.1 Evolução da Bacia: Superseqüências, Formações e suas $\begin{array}{ll}\text { características físicas } & 37\end{array}$

3.4 Pedologia 42

4 Investigações Geotécnicas 51

4.1 Situação 51

4.2 Diretrizes para programa de investigações geotécnicas 53

4.3 Conclusões sobre o estudo gelógico e geotécnico 61

4.4 Normatização 61

5 Torres, Terrenos e Fundações - Conceitos Gerais 62

5.1 Torres 62

$\begin{array}{lll}5.2 & \text { Terrenos } & 63\end{array}$ 
5.3 Fundações

5.3.1 Fundações aplicáveis a estruturas autoportantes (FURNAS, 2003)70

5.3.2 Fundações aplicáveis a estruturas estaiadas (FURNAS, 2003) 73

6 Proposta dos Conjuntos Torre x Terreno x Fundação 75

6.1 Conjuntos torre $x$ terreno $x$ fundação 75

6.1.1 Torre 76

$\begin{array}{lll}6.1 .2 & \text { Terreno } & 78\end{array}$

$\begin{array}{ll}6.1 .3 \text { Fundação } & 79\end{array}$

6.2 Comentários finais sobre os conjuntos torre $x$ terreno $x$ fundação 92

7 Confiabilidade das Fundações das Torres em LT's 94

7.1 Situação 94

7.2 Função de estado limite 96

7.3 Modelagem da Solicitação 96

7.3.1 Ação de vento segundo NBR5422 97

7.3.2 Esforços Globais 99

7.3.3 Modelo probabilístico para a Velocidade Básica do Vento 101

7.4 Modelagem da Resistência 101

$\begin{array}{ll}\text { 7.4.1 Equação de Terzagui } & 102\end{array}$

7.4.2 Modelo Probabilístico para as Propriedades do Solo 103

$\begin{array}{ll}7.5 \text { Rotinas Computacionais } & 104\end{array}$

7.6 Avaliação da Probabilidade de Falha e do Risco para fundações existentes na Bacia do Prata 107

$\begin{array}{ll}7.7 \text { Estudo Paramétrico } & 114\end{array}$

$\begin{array}{lll}7.8 & \text { Sensibilidade da probabilidade de falha } & 116\end{array}$

8 Síntese do Procedimento de Metodização e Constatações Particulares 119

8.1 Visão geral 119

8.2 Constatações particulares e cenários 120

8.2.1 Cenário determinístico 120

8.2.2 Cenário não-determinístico 121

8.3 Trabalhos complementares 123 
$\begin{array}{ll}\text { Anexo A } & 129\end{array}$

Linhas de transmissão na porção brasileira da Bacia do Prata 129

Expansão prevista das Linhas de transmissão na porção brasileira da Bacia do Prata

$\begin{array}{ll}\text { Anexo B } & 137\end{array}$

Confiabilidade Estrutural $\quad 137$

Estado Limite e Coeficientes de Segurança 137

O Problema Básico da Confiabilidade Estrutural 138

Métodos Analíticos 140

FORM ("First Order Reliability Method") 141

SORM ("Second Order Reliability Method") 142

Programa para análise de confiabilidade: FERUM 143

$\begin{array}{ll}\text { Apêndice A } & 146\end{array}$

Sondagens Geofísicas $\quad 146$

$\begin{array}{ll}\text { Apêndice B } & 148\end{array}$

$\begin{array}{ll}\text { Normatização } & 148\end{array}$

$\begin{array}{ll}\text { Apêndice C } & 150\end{array}$

NBR5422 150

$\begin{array}{ll}\text { Apêndice D } & 153\end{array}$ 


\section{Lista de figuras}

Figura 2.1 - Relação gráfica entre as bacias hidrográficas e os estados brasileiros.......24

Figura 2.2 - Mapa do Sistema de Transmissão Eletrosul (eletrosul.gov.br)....................25

Figura 2.3 - Mapa do Sistema de Transmissão Paulista (cteep.com.br)........................26

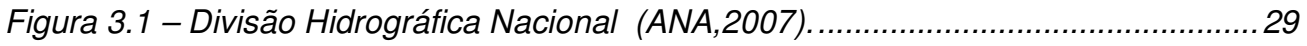

Figura 3.2 - Regiões hidrográficas, sub-regiões e unidades hidrográficas de referência

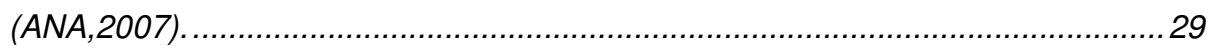

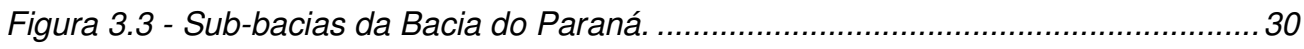

Figura 3.4 - Rios da porção brasileira da Bacia do Prata. ............................................ 31

Figura 3.5 - Sub-bacias da Bacia do Uruguai. ............................................................... 32

Figura 3.6 - Relevo do estado do Paraná (mapas.ibge.gov.br). ...................................... 33

Figura 3.7 - Relevo do estado de Santa Catarina (mapas.ibge.gov.br)............................ 33

Figura 3.8 - Relevo do estado do Rio Grande do Sul (mapas.ibge.gov.br)...................... 34

Figura 3.9 - Relevo do estado do Mato Grosso do Sul (mapas.ibge.gov.br).................... 34

Figura 3.10 - Relevo do estado de São Paulo (mapas.ibge.gov.br)................................ 35

Figura 3.11 - Relevo do estado de Minas Gerais (mapas.ibge.gov.br). .......................... 35

Figura 3.12 - Relevo do estado de Goiás (mapas.ibge.gov.br)..................................... 36

Figura 3.13 - Mapa de localização da Bacia do Paraná (Silva, 2006)............................. 37

Figura 3.14 - Mapa geológico simplificado da Bacia do Prata, seus principais elementos tectônicos e agentes geográficos (Silva, D.R.A, 2006)...................................... 38

Figura 3.15 - Carta estratigráfica da Bacia do Paraná (Silva, D.R.A, 2006).................... 42

Figura 3.16 - Pedologia do Rio Grande do Sul - IBGE. ............................................ 44

Figura 3.17 - Pedologia de Santa Catarina - IBGE. .................................................... 44

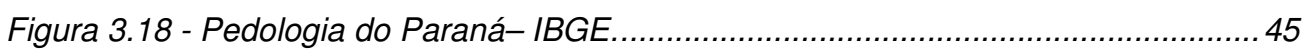

Figura 3.19 - Pedologia do Mato Grosso do Sul - IBGE. .......................................... 45

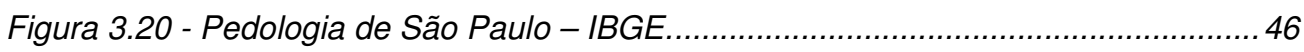

Figura 3.21 - Pedologia de Minas Gerais - IBGE ................................................. 47

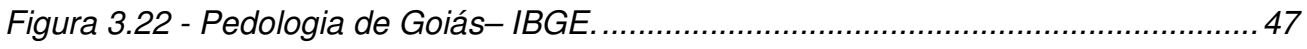

Figura 4.1 - Esquema para especificação da profundidade máxima de sondagens a

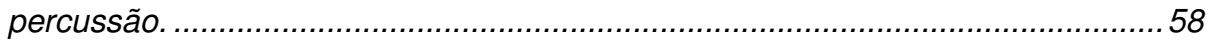

Figura 5.1 - Exemplos de torres: (a) autoportante; (b) estaiada (Garcia, 2005).............6 63

Figura 5.2 - Ligação entre a torre autoportante e a fundação em concreto, com materialização do ponto de aplicação dos esforços (Garcia, 2005). .....................67

Figura 5.3 - Exemplos de árvores de carregamento .............................................. 67

Figura 6.1 - Linhas de transmissão na Bacia do Prata (ons.gov.br).............................. 75

Figura 6.2 - Torre autoportante - Fluxo Engenharia.................................................. 77

Figura 6.3 - Torre estaiada - Fluxo Engenharia.................................................... 77 
Figura 6.4 - Fundação em sapata da torre autoportante de suspensão na LT Chavantes-

Botucatu.

Figura 6.5 - Armadura da fundação em sapata da torre autoportante de suspensão na

LT Chavantes-Botucatu.

Figura 6.6 - Fundação em sapata para torre autoportante de suspensão na LT Salto

Santiago-Cascavel Oeste...

Figura 6.7 - Armadura da fundação-tipo em sapata para torre autoportante de suspensão na LT Salto Santiago-Cascavel Oeste.

Figura 6.8 - Elevação, forma e armação da fundação em tubulão para torre autoportante de suspensão na LT Chavantes-Botucatu.

Figura 6.9 - Fundação em estaca para torre autoportante de suspensão na $L T$ Chavantes-Botucatu.

Figura 6.10 - Armação da fundação em estaca para torre autoportante de suspensão na

LT Chavantes-Botucatu.

Figura 6.11 - Fundação em bloco ancorado para torre autoportante de suspensão na $L T$

Chavantes-Botucatu. 86

Figura 6.12 -Fundação em sapata para o mastro de torre estaiada de suspensão na $L T$ Salto Santiago-Cascavel Oeste.

Figura 6.13 - Armação da fundação em sapata para o mastro de torre estaiada de suspensão na LT Salto Santiago-Cascavel Oeste.

Figura 6.14 -Fundação em bloco ancorado para o mastro de torre estaiada de suspensão na LT Salto Santiago-Cascavel Oeste.

Figura 6.15 - Armação da fundação em bloco ancorado para o mastro de torre estaiada de suspensão na LT Salto Santiago-Cascavel Oeste.

Figura 6.16 - Fundação em tubulão para o mastro de torre estaiada de suspensão na $L T$ Salto Santiago-Cascavel Oeste.

Figura 6.17 - Armação da fundação em tubulão para o mastro de torre estaiada de suspensão na LT Salto Santiago-Cascavel Oeste. 89

Figura 6.18 - Fundação em tubulão para estais de torre estaiada de suspensão na $L T$ Garabi-Itá. 90

Figura 6.19 - Fundação em bloco ancorado para estais de torre estaiada de suspensão na LT Garabi-Itá.

Figura 6.20 - Planta e detalhe da fundação em grelha para torre autoportante de suspensão na LT Ivaiporã-Itaberá-Tijuco Preto.

Figura 6.21 - Corte da fundação em grelha para torre autoportante de suspensão na $L T$ Ivaiporã-Itaberá-Tijuco Preto.

Figura 6.22 - Detalhe e Corte da fundação em grelha para torre autoportante de suspensão na LT Ivaiporã-Itaberá-Tijuco Preto. 92

Figura 7.1 - Ângulo de incidência do vento (NBR5422). 99

Figura 7.2 -Tensões na base da fundação. 100 


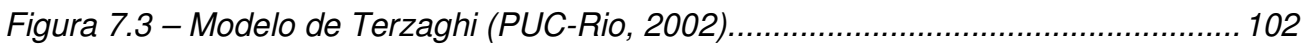

Figura 7.4 - Probabilidades de falha para fundações existentes na Bacia do Prata. ... 109

Figura 7.5 - Risco associado ao custo de reposição das estruturas.............................. 111

Figura 7.6 - Risco associado à falha por dia do sistema elétrico................................. 112

Figura 7.7 - Resultados do estudo paramétrico. .................................................. 115

Figura 7.8 - Gráfico comparativo dos fatores de importância. .................................... 117 


\section{Lista de tabelas}

Tabela 4.1 - Aplicação da metodologia de investigação geotécnica nas diversas fases de projeto para a implantação de uma $L T$ (ABGE, 1998)...................................... 54

Tabela 5.1 - Limites para os parâmetros geotécnicos dos solos...................................64

Tabela 5.2 - Limites para os parâmetros geotécnicos das rochas...................................64

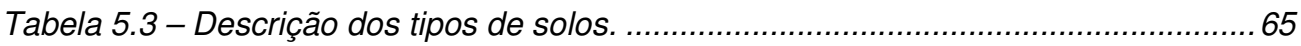

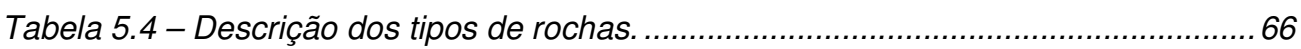

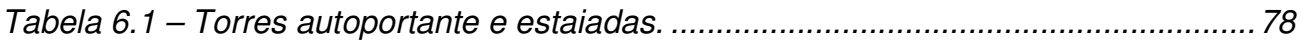

Tabela 6.2 - Exemplos de classificação dos tipos de solos dos projetos de fundação de

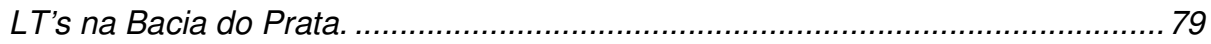

Tabela 6.3 - Conjunto torre $x$ terreno x fundação para o exemplo de torre estaiada tipo

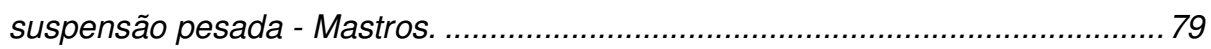

Tabela 6.4 - Conjunto torre $x$ terreno x fundação para o exemplo de torre estaiada tipo

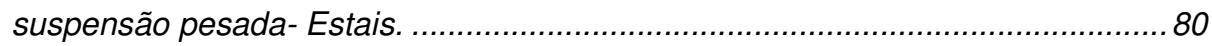

Tabela 6.5 - Conjunto torre $x$ terreno x fundação para o exemplo de torre autoportante

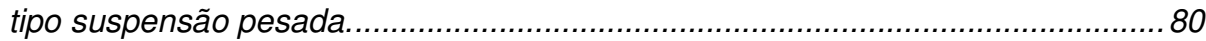

Tabela 7.1 - Exemplos avaliados de fundações existentes em torres de suspensão na

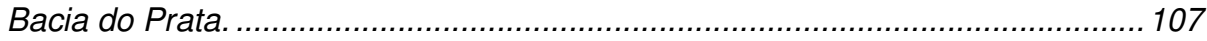

Tabela 7.2 - Variáveis aleatórias, médias e coeficientes de variação. .......................... 108

Tabela 7.3 - Análise de risco dos exemplos avaliados de fundações existentes na Bacia

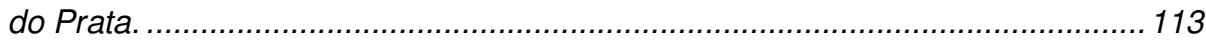

Tabela 7.4 - Fatores de importância para as fundações em sapata com $h_{e}=3,0 \mathrm{~m}$...116 


\section{Lista de símbolos}

A - área da fundação, em $\mathrm{m}^{2}$

ALT - altitude média da região de implantação da linha, em m

$A_{t}$ - esforço horizontal do vento, em $\mathrm{N}$

B - largura da fundação corrida na Teoria de Terzaghi

$\mathrm{C}$ - coesão, em $\mathrm{kN} / \mathrm{m}^{2}$

$\mathrm{C}_{\max }$ - força de compressão máxima, em $\mathrm{kN}$

$\mathrm{C}_{\mathrm{xT} 1}$ e $\mathrm{C}_{\mathrm{xT} 2}$ - coeficiente de arrasto próprio das faces 1 e 2

D - profundidade de assentamento da fundação corrida na Teoria de Terzaghi

$\mathrm{E}[\mathrm{G}(\mathrm{X})]$ - valor esperado da função de falha

$\operatorname{Var}[\mathrm{G}(\mathrm{X})]$ - variância da função de falha

$\mathrm{F}_{\mathrm{R}}($.$) - função de distribuição acumulada de probabilidades$

$\mathrm{F}_{\mathrm{X}}(\mathrm{x})$ - função de distribuição acumulada de probabilidades

$G(X)$ - função de desempenho ou função de falha

$\mathrm{H}_{\mathrm{mi}}$ - altura do ponto médio do módulo i da torre ao nível do terreno, em $\mathrm{m}$

$\mathrm{H}_{\mathrm{t}}$ - altura total da torre, em $\mathrm{m}$

$\mathrm{K}_{\mathrm{d}}$ - relação entre os valores médios de vento a $10 \mathrm{~m}$ de altura do solo para diferentes períodos de integração e rugosidade de terrenos

$\mathrm{K}_{\mathrm{r}}$ - o coeficiente de rugosidade

$\mathrm{M}$ - momento global, em kNm

$\mathrm{N}$ - soma das cargas gravitacionais, em $\mathrm{kN}$

$\mathrm{N}_{\mathrm{i}}$ - fatores de capacidade de carga na equação de Terzaghi

$\mathrm{P}_{\mathrm{f}}$ - probabilidade de falha

$\mathrm{P}[$.$] - probabilidade$

Q - carregamento

$\mathrm{R}$ - resistência ou capacidade

$\mathrm{S}$ - solicitação, demanda ou carregamento

$\mathrm{S}_{\mathrm{T} 1}$ e $\mathrm{S}_{\mathrm{T} 2}$ - área líquida total de cada face projetada ortogonalmente sobre o plano

vertical situado na direção das faces 1 e $2, \mathrm{em} \mathrm{m}^{2}$

$\mathrm{T}$ - período de retorno

$\mathrm{T}_{\max }$ - força de tração máxima, em kN 
U - vetor de variáveis normais não correlacionadas

$\mathrm{U}^{*}$ - ponto de projeto

$\mathrm{Vb}$ - velocidade básica do vento, em $\mathrm{m} / \mathrm{s}$

$\mathrm{Vp}$ - velocidade do vento de projeto, em $\mathrm{m} / \mathrm{s}$

W - módulo de resistência da fundação, em $\mathrm{m}^{3}$

$\mathrm{X}$ - vetor das variáveis originais

$\mathrm{b}$ - menor dimensão da base da fundação, em m

$d_{p}$ - distância entre as pernas da torre na direção da ação do vento

$\mathrm{f}_{\mathrm{R}, \mathrm{S}}(\mathrm{r}, \mathrm{s})$ - função de densidade conjunta das variáveis $\mathrm{R}$ e $\mathrm{S}$

$\mathrm{f}_{\mathrm{S}}(\mathrm{s})$ - função densidade de probabilidade da variável $\mathrm{S}$

$\mathrm{f}_{\mathbf{X}}(\mathrm{x})$ - função densidade de probabilidade

$\mathrm{h}_{\mathrm{e}}$ - profundidade de assentamento das fundações

hi - altura do ponto médio da cada módulo da torre ao ponto equivalente à

profundidade de assentamento, em $\mathrm{m}$

$\mathrm{h}(\mathrm{u})$ - função de linearização

1 - comprimento do módulo da torre

m - número total de módulos da torre

$\mathrm{m}_{\mathrm{i}}-$ módulo i da torre

n - parâmetro dependente da rugosidade do terreno e do período de integração

$\mathrm{q}_{0}$ - pressão dinâmica de referência

$\mathrm{q}_{\mathrm{ult}}$ - capacidade de suporte da fundação na ruptura, em kN

$\mathrm{s}_{\mathrm{i}}$ - fatores de forma na equação de Terzaghi

$\mathrm{t}$ - temperatura, em ${ }^{\circ} \mathrm{C}$

$\alpha$ - vetor normal à superfície

$\alpha$ - ângulo de arrancamento, $\mathrm{em}^{\mathrm{o}}$

$\beta$ - índice de confiabilidade

$\delta$ - coeficiente de variação

$\phi$ - ângulo de atrito, em ${ }^{\circ}$

$\gamma$ - peso específico, em $\mathrm{kN} / \mathrm{m}^{3}$

$\kappa_{\mathrm{i}}$ - curvaturas principais da superfície de falha do ponto de projeto

$\mu_{\mathrm{G}}$ - valor médio da função $\mathrm{G}$ 
$\rho$ - massa específica do ar, em $\mathrm{kg} / \mathrm{m}^{3}$

$\sigma_{\mathrm{G}}-$ desvio padrão da função $\mathrm{G}$

$\sigma_{\text {Nglobal }}$ - tensão normal global

$\theta$ - ângulo de incidência do vento 